\title{
TRANSITIVITY, TWO-SIDED LIMIT SHADOWING PROPERTY AND DENSE $\omega$-CHAOS
}

\author{
Piotr Oprocha
}

\begin{abstract}
We consider $\omega$-chaos as defined by S. H. Li in 1993. We show that c-dense $\omega$-scrambled sets are present in every transitive system with two-sided limit shadowing property (TSLmSP) and that every transitive map on topological graph has a dense Mycielski $\omega$-scrambled set. As a preliminary step, we provide a characterization of dynamical properties of maps with TSLmSP.
\end{abstract}

\section{Introduction}

In 1993, S. H. Li introduced in [15] the notion of $\omega$-chaos for interval maps. Since then, only a small progress was made in the topic. The authors of [29] show that transitive map on the unit interval has a dense Mycielski $\omega$-scrambled set, and later Bobok presented in [5] how this result can be extended to produce a map on the unit $n$-dimensional cube with $\omega$-scrambled set. A few special cases of construction of uncountable $\omega$-scrambled sets for systems on Cantor set are dealt in [13, 24] (see also [15] and [29]) and not much more beyond these results is known. The main difficulty is that standard techniques working for residual relations (an effective tool for most of definitions of chaos stated in terms of pairs or tuples) cannot be employed here. This makes proving $\omega$-chaos in concrete cases a quite challenging problem.

In this paper we use two tools when proving $\omega$-chaos. First of all, we extend results of $[29]$ to show that on topological graphs transitivity implies $\omega$-chaos (with dense Mycielski $\omega$-scrambled set), provided that entropy is positive (or equivalently, the map is not an irrational relation on the unit circle). Our construction highly rely on the recent results from [10] (and of course, some ideas from [29]).

Second tool, applicable in a more general case is two-sided limit shadowing property (abbrev. TSLmSP). This property was probably first introduced by Pilyugin in his book [25] and is a version of standard shadowing property (SP

Received December 14, 2013.

2010 Mathematics Subject Classification. Primary 37B05; Secondary 37C50, 37B20.

Key words and phrases. shadowing property, limit shadowing, pseudo-orbit, asymptotic tracing, minimal set, $\omega$-chaos, topological graph. 
for short), where emphasis is put on asymptotic aspects of tracing. It can be proved that all structurally stable diffeomorphisms exhibit TSLmSP, while the class of diffeomorphisms with TSLmSP is larger (see Theorems 2 and 3 in [26]). Sakai proved in [27] that $C^{1}$-generic diffeomorphism has a combination of SP and TSLmSP, called s-limit shadowing property, if and only if it satisfies Axiom A and no-cycle condition (see [27] for more details). It was later proved in [17] that for $C^{1}$-generic diffeomorphism the above condition is actually equivalent to TSLmSP (called weak shadowing property in [17]). Carvahlo characterized in [6] the $C^{1}$-interior of the set of diffeomorphisms with TSLmSP on closed manifolds as exactly the set of transitive Anosov diffeomorphisms. In the case of expansive homeomorphism, shadowing property and TSLmSP are mutually equivalent properties [14]. However, it is not hard to verify (e.g. taking an infinite Cartesian product) that TSLmSP can exist in systems which are not expansive. It is also a consequence of results of [26] mentioned above. Beyond the above mentioned results, not much more is known about TSLmSP. Recently, an interesting connection between a modified version of TSLmSP (so-called two-sided limit shadowing property with a gap) and various mixing properties for homeomorphism has been addressed in [7].

This paper is organized as follows. The next section collects a few most important facts and definitions. As we could see in previous paragraph, the most work done on TSLmSP was devoted to special classes of diffeomorphisms. In particular, no general properties of maps with TSLmSP were studied. To fill this gap in the literature, first we prove in Section 3 a few theorems for a better understanding of dynamics of maps with TSLmSP (e.g. a kind of Smale and Bowen spectral decompositions). Next we prove that $\omega$-chaos can be a consequence of TSLmSP. In the last section of the paper we show that on topological graphs transitivity (no shadowing assumption) is enough to induce $\omega$-chaos with dense Mycielski $\omega$-scrambled set.

\section{Preliminaries}

Denote by $\mathbb{N}$ the set of positive integers, by $\mathbb{Z}$ the set of integers and $\mathbb{N}_{0}=$ $\mathbb{N} \cup\{0\}$. A set $Q \subset X$ is $c$-dense if for any nonempty open set $U$ the set $U \cap Q$ is uncountable. A point $x \in A$ is isolated (in $A$ ) if there is an open neighborhood $U$ of $x$ such that $U \cap A=\{x\}$. A set is prefect if it is nonempty, closed and does not have isolated points. If there is a basis of clopen (i.e., closed and open at the same time) sets, then we say that $X$ is zero dimensional. A set $A$ is Cantor if it is perfect and zero dimensional and Mycielski if it can be presented as a countable union of Cantor sets.

A pair $(X, f)$ is a dynamical system if it consists of a compact metric space $(X, d)$ and a continuous map $f: X \rightarrow X$. We denote the (positive) orbit of a point $x \in X$ by $\operatorname{Orb}^{+}(x, f)=\left\{x, f(x), f^{2}(x), \ldots\right\}$ and an $\omega$-limit set of $x$ is defined by $\omega(x, f)=\bigcap_{n=1}^{\infty} \overline{\operatorname{Orb}^{+}\left(f^{n}(x), f\right)}$. A set $M$ is minimal if $M=$ $\overline{\mathrm{Orb}^{+}(x, f)}$ for every $x \in M$. 
The following lemma is a special case of theorem proved by Mycielski in [19]. Result of [19] is a more powerful tool, however we do not need it here in such generality.

Lemma 1. Let $X$ be a compact perfect set. If $R$ is a residual subset of $X \times X$, then there exists a dense Mycielski set $M \subset X$ such that $M \times M \subset R \cup \Delta$.

\subsection{Shadowing}

Let $\delta>0$ be a positive constant. A sequence $\left\{y_{n}\right\}_{n \in \mathbb{Z}} \subset X$ is a $\delta$-pseudoorbit of $f$ if

$$
d\left(f\left(y_{n}\right), y_{n+1}\right) \leq \delta \text { for every } n \in \mathbb{Z} .
$$

Note that 0-pseudo-orbit of $f$ is simply its genuine (full) orbit. A $\delta$-pseudo-orbit $\left\{y_{n}\right\}$ is two-sided limit $\delta$-pseudo-orbit of $f$ if it satisfies

$$
\lim _{|n| \rightarrow \infty} d\left(f\left(y_{n}\right), y_{n+1}\right)=0 .
$$

If only condition (1) is satisfied (i.e., no particular $\delta>0$ is established), we will say that $\left\{y_{n}\right\}_{n \in \mathbb{Z}}$ is a two-sided limit pseudo-orbit of $f$.

We say that $f$ posses the shadowing property ( $S P$ for short) if for every $\varepsilon>0$ there exists $\delta>0$ satisfying the following condition: given a $\delta$-pseudo-orbit $\left\{y_{n}\right\}_{n \in \mathbb{Z}}$ we can find a corresponding 0-pseudo-orbit $\left\{x_{n}\right\}_{n \in \mathbb{Z}}$ which $\varepsilon$-traces $\left\{y_{n}\right\}_{n \in \mathbb{Z}}$, i.e.,

$$
d\left(x_{n}, y_{n}\right) \leq \varepsilon \text { for every } n \in \mathbb{Z}
$$

If there is $\beta>0$ such that for any two-sided limit $\beta$-pseudo-orbit $\left\{y_{n}\right\}_{n \in \mathbb{Z}}$ we can find a 0 -pseudo-orbit $\left\{x_{n}\right\}_{n \in \mathbb{Z}}$ which traces $\left\{y_{n}\right\}_{n \in \mathbb{Z}}$ in the limit sense, i.e.,

$$
\lim _{|n| \rightarrow \infty} d\left(x_{n}, y_{n}\right)=0
$$

then we say that $f$ possess the two-sided limit shadowing property (abbrev. TSLmSP).

Sometimes it is more convenient to work with finite pseudo-orbits when speaking about SP. A finite sequence $x_{0}, \ldots, x_{n}$ is a (finite) $\delta$-pseudo-orbit (or a $\delta$-chain from $x_{0}$ to $\left.x_{n}\right)$ if $d\left(f\left(x_{i}\right), x_{i+1}\right)<\delta$ for $i=0, \ldots, n-1$. A point $z$ is $\varepsilon$-tracing a pseudo-orbit $\left\{x_{i}\right\}_{i=0}^{n}$ if $d\left(f^{i}(z), x_{i}\right)<\varepsilon$ for $i=0, \ldots, n$. When $X$ is compact and $(X, f)$ is onto, it is not hard to verify that $(X, f)$ has SP if and only if for every $\varepsilon>0$ there is $\delta>0$ such that every finite $\delta$-pseudo-orbit is $\varepsilon$-traced by a point in $X$.

Fix $x, y \in X$. If for every $\delta>0$ there are finite $\delta$-pseudo-orbits from $x$ to $y$ and from $y$ to $x$, then we write $x \sim y$. A point $x$ is chain-recurrent if $x \sim x$. The set of all chain recurrent points is denoted $C R(f)$. It is not hard to see that $\sim$ is an equivalence relation on $C R(f)$. If $x \in C R(f)$, then $[x]_{\sim}$ is well defined, and we call it a chain recurrent class (of $x$ ). 
Given a 0 -pseudo-orbit $\left\{x_{n}\right\}_{n \in \mathbb{Z}}$ we define

$$
\omega\left(\left\{x_{n}\right\}_{n \in \mathbb{Z}}, f\right)=\bigcap_{k=1}^{\infty} \overline{\left\{x_{n}: n>k\right\}} \text { and } \alpha\left(\left\{x_{n}\right\}_{n \in \mathbb{Z}}, f\right)=\bigcap_{k=1}^{\infty} \overline{\left\{x_{-n}: n>k\right\}} .
$$

Note that $\omega\left(\left\{x_{n}\right\}_{n \in \mathbb{Z}}, f\right)=\omega\left(x_{k}, f\right)$ for every $k \in \mathbb{Z}$.

\subsection{Transitivity, equicontinuity and $\omega$-chaos}

A dynamical system $(X, f)$ is equicontinuous if for every $\epsilon>0$, there is $\delta>0$ with the property that $d(x, y)<\delta$ implies $d\left(f^{n}(x), f^{n}(y)\right)<\epsilon$ for every $x, y \in X$ and every $n \in \mathbb{N}$. A point $x \in X$ is equicontinuous if for every $\epsilon>0$, there is $\delta>0$ with the property that $d(x, y)<\delta$ implies $d\left(f^{n}(x), f^{n}(y)\right)<\epsilon$ for every $y \in X$ and every $n \in \mathbb{N}$. By compactness of $X$, a system $(X, f)$ is equicontinuous if and only if every point in $X$ is equicontinuous.

A dynamical system $(X, f)$ is sensitive if there exists $\delta>0$ such that for any nonempty open subset $U$ of $X$, we have $\operatorname{diam}\left(f^{n}(U)\right)>\delta$ for some $n \in \mathbb{N}$. A point $x \in X$ is sensitive if it is not equicontinuous, that is, there exists a $\delta>0$ such that for any neighborhood $U$ of $x$ there is $n>0$ such that $\operatorname{diam}\left(f^{n}(U)\right)>\delta$. Clearly, if $(X, f)$ is sensitive, then every $x \in X$ is sensitive.

The following definition of $\omega$-chaos was introduced by S. H. Li in [15].

Definition 2. A set $S \subset X$ containing at least two points is called an $\omega$ scrambled set for $f$ if, for any two $x \neq y$ in $S$,

(1) $\omega_{f}(x) \backslash \omega_{f}(y)$ is uncountable,

(2) $\omega_{f}(x) \cap \omega_{f}(y) \neq \emptyset$ and

(3) $\omega_{f}(x) \backslash \operatorname{Per}(f) \neq \emptyset$.

The map $f$ is $\omega$-chaotic if there is an uncountable $\omega$-scrambled set. By an $\omega$-chaotic pair we mean any $\omega$-scrambled set of cardinality 2 .

In minimal set every point has dense orbit. It is not hard to verify that if a minimal set $M$ has an isolated point, then $M$ is a single periodic orbit, and if $M$ does not have isolated points, then it is uncountable, as is every perfect compact set.

Remark 3. In most of the known constructions it is possible to replace conditions (2), (2) by the following stronger condition:

$\left(1^{\prime}\right) \omega_{f}(x) \backslash \omega_{f}(y)$ contains an infinite minimal set.

In this paper, when we prove $\omega$-chaos, we construct $\omega$-scrambled sets satisfying this stronger condition.

\subsection{Subshifts}

We denote $\Sigma_{2}^{+}=\{0,1\}^{\mathbb{N}}$ endowed with the metric $d(x, y)=2^{-k}$ when $x \neq y$, where $k=\min \left\{i: x_{i} \neq y_{i}\right\}$. The space $\left(\Sigma_{2}^{+}, d\right)$ is compact and the shift map $\sigma: \Sigma_{2}^{+} \rightarrow \Sigma_{2}^{+}$, given by $\sigma(x)_{i}=x_{i+1}$ for all $i \in \mathbb{N}$, is continuous. For $x \in \Sigma_{2}^{+}$we usually write $x=x_{1} x_{2} x_{3} \ldots$ instead of $x=\left(x_{1}, x_{2}, x_{3}, \ldots\right)$. We denote by $0^{\infty}$ 
the constant sequence $0^{\infty}=0000 \cdots$. By a shift or subshift we mean any closed and $\sigma$-invariant subset of $\Sigma_{2}^{+}$. For $1 \leq i \leq j$ we denote $x_{[i, j]}=x_{i} x_{i+1} \cdots x_{j}$ and $x_{[i, j)}=x_{[i, j-1]}$. The set of words of length $n$ in a subshift $X$ is denoted $B_{n}(X)=\left\{x_{[i, i+n)}: x \in X, i \in \mathbb{N}\right\}$.

\section{TSLmSP and transitivity}

We say that regularly closed sets $D_{0}, \ldots, D_{n-1}$ (i.e., $\overline{\operatorname{Int} D_{i}}=D_{i}$ ) form a periodic decomposition of $X$ if they cover $X, D_{i} \cap \operatorname{Int} D_{j}=\emptyset$ for $i \neq j$, and $f\left(D_{i}\right)=D_{i+1}$ for $i=0, \ldots, n-1$, where for technical reasons we put $D_{n}=D_{0}$.

The proof of the following fact is standard. We present it for completeness.

Lemma 4. Let $D_{0}, \ldots, D_{n-1}$ form a periodic decomposition for a dynamical system $(X, f)$ and assume that $\left(D_{i}, f^{n}\right)$ is transitive for every $i$. If $(X, f)$ has $S P$, then $D_{i} \cap D_{j}=\emptyset$ for $i \neq j$.

Proof. Suppose that there are $i \neq j$ such that $D_{i} \cap D_{j} \neq \emptyset$. By the definition of decomposition $D_{i}=\overline{\operatorname{Int} D_{i}}$, hence Int $D_{i} \neq \emptyset$. Recall also that Int $D_{i} \cap D_{j}=\emptyset$.

Fix points $z_{1} \in \operatorname{Int} D_{i}, z_{2} \in \operatorname{Int} D_{j}$ and take $\varepsilon>0$ such that $B\left(z_{1}, 2 \varepsilon\right) \subset$ Int $D_{i}, B\left(z_{2}, 2 \varepsilon\right) \subset$ Int $D_{j}$. Let $\delta$ be provided to $\varepsilon$ by SP.

Suppose that there is $z \in D_{i} \cap D_{j}$. By transitivity of $f^{n}$ on $D_{i}$ and on $D_{j}$ there are finite $\delta$-pseudo-orbits $\gamma, \xi$ of length $|\gamma|=s n,|\xi|=t n+1$ for some $s, t>$ 0 , such that $\gamma_{1}=z_{1}, d\left(f\left(\gamma_{s n}\right), z\right)<\beta / 2$ and $d\left(\xi_{1}, z\right)<\beta / 2, \xi_{t n+1}=z_{2}$. If $x$ is a point which is $\varepsilon$-tracing the $\delta$-pseudo-orbit $\zeta=\gamma \xi$ formed by concatenation of $\gamma$ with $\xi$, then $d\left(x, z_{1}\right)<\varepsilon$ and $d\left(f^{(t+s) n}, \zeta_{(t+s) n+1}\right)=d\left(f^{(t+s) n}, \xi_{t n+1}\right)<\varepsilon$. This immediately implies that

$$
f^{(s+t) n}(x) \in f^{(s+t) n}\left(\operatorname{Int} D_{i}\right) \cap \operatorname{Int} D_{j} \subset D_{i} \cap \operatorname{Int} D_{j}=\emptyset .
$$

This is a contradiction, which finishes the proof.

Lemma 5. If $(X, f)$ is a transitive dynamical system with TSLmSP, then there is $N>0$ such that if $D_{0}, \ldots, D_{n-1}$ is a periodic decomposition of $X$, then $n \leq N$.

Proof. Let $\beta$ be provided by TSLmSP. Let $U_{1}, \ldots, U_{N}$ be a cover of $X$ by open sets of diameter smaller than $\beta / 3$. If $D_{0}, \ldots, D_{n}$ is a periodic decomposition, and $n \geq N$, then there are $i \neq j$ and $k$ such that $D_{i} \cap U_{k} \neq \emptyset$ and $D_{j} \cap U_{k} \neq \emptyset$. We will repeat argument from the proof Lemma 4 to obtain that Int $D_{i} \cap D_{j} \neq \emptyset$ which will lead to a contradiction.

Take recurrent points $z_{1} \in \operatorname{Int} D_{i} \cap U_{k}$ and $z_{2} \in \operatorname{Int} D_{j} \cap U_{k}$. It is well known that point recurrent for $f$ is also recurrent for $f^{n}$. Therefore, there are increasing sequences $r_{i}, s_{i}$ such that $d\left(f^{n r_{i}}\left(z_{1}\right), z_{1}\right)<\beta 2^{-i}$ and $d\left(f^{n s_{i}}\left(z_{2}\right), z_{2}\right)<\beta 2^{-i}$. Let $\gamma_{i}=\left\{z_{1}, f\left(z_{1}\right), \ldots, f^{n r_{i}-1}\left(z_{1}\right)\right\}$ and $\xi_{i}=\left\{z_{2}, f\left(z_{2}\right), \ldots, f^{n s_{i}-1}\left(z_{2}\right)\right\}$ and consider the sequence obtained as the following two-sided infinite concatenation

$$
\zeta=\bigcup_{n=-\infty}^{1}\left\{\gamma_{n}\right\} \cup \bigcup_{n=1}^{\infty}\left\{\xi_{n}\right\}=\cdots \gamma_{n} \cdots \gamma_{2} \gamma_{1} \xi_{1} \xi_{2} \cdots \xi_{n} \cdots
$$


Note that $\zeta$ is an limit $\beta$-pseudo orbit because

$$
d\left(f\left(f^{n r_{1}-1}\left(z_{1}\right)\right), z_{2}\right) \leq d\left(f^{n r_{1}}\left(z_{1}\right), z_{1}\right)+d\left(z_{1}, z_{2}\right)<\beta / 2+\beta / 3<\beta .
$$

Let $\left\{x_{n}\right\}_{n \in \mathbb{Z}}$ be a 0 -pseudo-orbit provided to $\zeta$ by TSLmSP. Note that both sets $\left\{l>0: \zeta_{-n l}=z_{1}\right\}$ and $\left\{t>0: \zeta_{n t}=z_{2}\right\}$ are infinite. Hence there are $l, t>0$ such that $y=x_{-l n} \in B\left(z_{1}, \varepsilon\right)$ and $x_{t n} \in B\left(z_{2}, \varepsilon\right) \subset$ Int $D_{j}$, which in turn implies that

$$
f^{(t+l) n}(y)=x_{t n} \in f^{(t+l) n}\left(D_{i}\right) \cap \operatorname{Int} D_{j}=\emptyset .
$$

This is a contradiction, hence the proof is finished.

Lemma 6. If $(X, f)$ has TSLmSP, then there are finitely many chain recurrent classes, that is $C R(f)=\left[x_{1}\right]_{\sim} \cup \cdots \cup\left[x_{n}\right]_{\sim}$ for some points $x_{1}, \ldots, x_{n}$.

Proof. Assume that there are infinitely many chain-recurrent classes. Since $[x]_{\sim}$ is closed for each $x \in C R(f)$ there is a sequence of points $\left\{x_{i}\right\}_{i=1}^{\infty}$ such that all $\left[x_{i}\right]_{\sim}$ are distinct and are convergent in the Hausdorff metric $\mathcal{H}_{d}$ induced by $d$ onto the space of all nonempty compact subsets of $X$. In particular $\left\{\left[x_{i}\right]_{\sim}\right\}_{i=1}^{\infty}$ is a Cauchy sequence in the Hausdorff metric. Let $\beta>0$ be provided by TSLmSP. There are distinct $x, y \in\left\{x_{i}\right\}_{i=1}^{\infty}$ with

$$
\operatorname{dist}\left([x]_{\sim},[y]_{\sim}\right) \leq \mathcal{H}_{d}\left([x]_{\sim},[y]_{\sim}\right)<\beta .
$$

Therefore, without loss of generality (replacing $x, y$ by other points in their equivalence classes if necessary) we may assume that $d(x, y)<\beta$. But $x, y \in$ $C R(f)$, hence we can easily construct a two-sided limit $\beta$-pseudo-orbit $\xi=$ $\left\{\xi_{i}\right\}_{i \in \mathbb{Z}}$ such that $\left\{\xi_{i}\right\}_{i \geq 0} \subset[y]_{\sim},\left\{\xi_{i}\right\}_{i<0} \subset[x]_{\sim}$ and both sets $\left\{i<0: \xi_{i}=x\right\}$ and $\left\{i>0: \xi_{i}=y\right\}$ are infinite. Then as an immediate consequence of TSLmSP we obtain that for every $\varepsilon>0$ there is an $\varepsilon$-chain from $x$ to $y$. Similarly, we can construct two-sided limit $\beta$-pseudo-orbit $\gamma=\left\{\gamma_{i}\right\}_{i \in \mathbb{Z}}$ such that $\left\{\gamma_{i}\right\}_{i<0} \subset[y]_{\sim}$, $\left\{\xi_{i}\right\}_{i>0} \subset[x]_{\sim}$ and both sets $\left\{i>0: \xi_{i}=x\right\}$ and $\left\{i<0: \xi_{i}=y\right\}$ are infinite. Consequently, for every $\varepsilon>0$ there is an $\varepsilon$-chain from $y$ to $x$. We have just proved that $x \sim y$ which is a contradiction.

Lemma 7. Suppose that $\Lambda$ is a chain-recurrent class and that $(\Lambda, f)$ has TSLmSP. Then $(\Lambda, f)$ is transitive.

Proof. Let $\beta>0$ be provided by TSLmSP. Fix any $x, y \in \Lambda$. Repeating arguments from the proof of Lemma 6 we can find a limit $\beta$-pseudo-orbit $\left\{x_{n}\right\}_{n \in \mathbb{Z}}$ such that both sets $\left\{i>0: \xi_{i}=y\right\}$ and $\left\{i<0: \xi_{i}=x\right\}$ are infinite. Let $\left\{z_{n}\right\}_{n \in \mathbb{Z}}$ be a 0 -pseudo-orbit which is tracing $\left\{x_{n}\right\}_{n \in \mathbb{Z}}$ in the limit sense. Then for every $N>0$ there are indexes $i, j>N$ such that $d\left(z_{-i}, x\right)<1 / N$ and $d\left(z_{j}, y\right)<1 / N$. This immediately implies that for every $\varepsilon>0$ and every $n \in \mathbb{Z}$ there are $\varepsilon$-chains from $x$ to $z_{n}$ and from $z_{n}$ to $y$. But $\Lambda$ is chain recurrent class, hence $x \sim y$ and therefore $x \sim z_{n}$ for every $n \in \mathbb{Z}$, in particular $\left\{z_{n}\right\}_{n \in \mathbb{Z}} \subset \Lambda$. But if we take any open sets $x \in U, y \in V$, then there are $n<m$ such that $z_{n} \in U, z_{m} \in V$, proving that $f^{m-n}(U) \cap V \neq \emptyset$. Indeed $(\Lambda, f)$ is transitive. 
Theorem 8. If $(X, f)$ has TSLmSP, then $\left.f\right|_{C R(f)}$ has shadowing and there are closed invariant sets $\Lambda_{1}, \ldots, \Lambda_{n}$ such that $C R(f)=\bigcup_{i=1}^{n} \Lambda_{i}$ and $\left.f\right|_{\Lambda_{i}}$ is transitive and has $S P$ for each $i=1, \ldots, n$. Furthermore, for every $i$ there is $m=m(i)$ and periodic decomposition $D_{0}, \ldots, D_{m-1}$ of $\Lambda_{i}$ such that sets $D_{j}$ are disjoint and $\left(D_{j}, f^{m}\right)$ is mixing for $j=0,1, \ldots, m-1$.

Proof. Let $\beta>0$ be provided by TSLmSP and let $\Lambda_{1}, \ldots, \Lambda_{n}$ be a partition of $C R(f)$ into chain-recurrent classes provided by Lemma 6 . Denote $\varepsilon=\frac{1}{2} \min \left\{\min _{i \neq j} \operatorname{dist}\left(\Lambda_{i}, \Lambda_{j}\right), \beta\right\}$. Clearly, if we take any two-sided limit $\varepsilon$-pseudo-orbit contained completely in $\Lambda_{i}$ and $\left\{x_{n}\right\}_{n \in \mathbb{Z}}$ is a full orbit (i.e., 0 -pseudo-orbit) which traces it in the limit sense, then $\omega\left(\left\{x_{n}\right\}_{n \in \mathbb{Z}}, f\right) \cap \Lambda_{i} \neq \emptyset$ and $\alpha\left(\left\{x_{n}\right\}_{n \in \mathbb{Z}}, f\right) \cap \Lambda_{i} \neq \emptyset$. But then, since $\Lambda_{i}$ is chain-recurrent class, we clearly have that $\left\{x_{n}\right\}_{n \in \mathbb{Z}} \subset \Lambda_{i}$. This shows that $\left(\Lambda_{i}, f\right)$ has TSLmSP for each $i$ and therefore is transitive by Lemma 7 . By results of [11] we obtain that $\left(\Lambda_{i}, f\right)$ has SP.

If $\left(\Lambda_{i}, f\right)$ is totally transitive, then it must be mixing (e.g. see [12]). Otherwise there exists a periodic decomposition of $\Lambda_{i}$ (see [3]), and by Lemma 5 length of every such decomposition is bounded. But then, again by results of [3], we obtain that there are sets $D_{0}, \ldots, D_{m-1}(m \geq 1)$ forming periodic decomposition of $\Lambda_{i}$ and such that $\left(D_{j}, f^{m}\right)$ is totally transitive. By Lemma 4 sets $D_{j}$ are disjoint, and hence it is clear that $\left(D_{j}, f^{m}\right)$ has SP. Repeating previous argument, we see that each $\left(D_{j}, f^{m}\right)$ is mixing completing the proof.

Corollary 9. If $(X, f)$ is a minimal system with TSLmSP, then it is a periodic orbit.

Proof. By results of [11] we obtain that $(X, f)$ has SP. Then it is a consequence of [18] that $(X, f)$ is equicontinuous and by Theorem 8 there is a periodic decomposition $D_{0}, \ldots, D_{n-1}$ of $X$ such that $\left(D_{i}, f^{n}\right)$ is mixing for every $i$. But the only possibility for equicontinuous system to be mixing is that $D_{i}$ is a singleton. The proof is finished.

\section{TSLmSP and $\omega$-scrambled sets}

Lemma 10. Let $(X, f)$ be a transitive dynamical system with TSLmSP. Let $M_{1}, M_{2}$ be two minimal sets. There exists $z$ and at most countable set $Z$ such that $\omega_{f}(z)=M_{1} \cup M_{2} \cup Z$.

Proof. Fix any $\varepsilon>0$ and let $\beta>0$ be provided by TSLmSP. By transitivity, there exist points $x, y \in X$ and integers $n, m>0$ such that $x, f^{m}(y) \in$ $B\left(M_{1}, \beta / 2\right)$ and $y, f^{n}(x) \in B\left(M_{2}, \beta / 2\right)$. Since $M_{1}, M_{2}$ are minimal, there are sequences $\xi=\left\{\xi_{i}\right\}_{i=-\infty}^{0} \subset M_{1}, \zeta=\left\{\zeta_{i}\right\}_{i=-\infty}^{0} \subset M_{2}$ and points $p \in M_{1}$, $q \in M_{2}$ such that $d\left(p, f^{m}(y)\right)<\beta, d\left(q, f^{n}(x)\right)<\beta, d\left(\xi_{0}, x\right)<\beta, d\left(\zeta_{0}, y\right)<\beta$ and $f\left(\xi_{i}\right)=\xi_{i+1}, f\left(\zeta_{i}\right)=\zeta_{i+1}$ for all $i<0$.

Note that the following two-sided sequences

$$
\phi=\left\{\phi_{n}\right\}_{n \in \mathbb{Z}}=\left\{\ldots, \xi_{-2}, \xi_{-1}, x, \ldots, f^{n-1}(x), q, f(q), f^{2}(q), \ldots\right\},
$$




$$
\psi=\left\{\psi_{n}\right\}_{n \in \mathbb{Z}}=\left\{\ldots, \zeta_{-2}, \zeta_{-1}, y, \ldots, f^{m-1}(y), p, f(p), f^{2}(p), \ldots\right\}
$$

form two-sided limit pseudo-orbits. By TSLmSP there are 0-pseudo-orbits $\hat{\phi}, \hat{\psi}$ (i.e., actual orbits) which are tracing $\phi$ and $\psi$, respectively, in the limit sense, i.e., $\lim _{|n| \rightarrow \infty} d\left(\phi_{n}, \hat{\phi}_{n}\right)=0$ and $\lim _{|n| \rightarrow \infty} d\left(\psi_{n}, \hat{\psi}_{n}\right)=0$. Since $\alpha\left(\left\{\hat{\phi}_{n}\right\}_{n \in \mathbb{Z}}, f\right)$ $=\alpha\left(\left\{\phi_{n}\right\}_{n \in \mathbb{Z}}, f\right)=M_{1}$ and $\omega\left(\left\{\hat{\psi}_{n}\right\}_{n \in \mathbb{Z}}, f\right)=\omega\left(\left\{\psi_{n}\right\}_{n \in \mathbb{Z}}, f\right)=M_{1}$, there is a decreasing sequence $\left\{k_{i}\right\}_{i=0}^{\infty} \subset(-\infty, 0)$ and an increasing sequence $\left\{\hat{k}_{i}\right\}_{i=0}^{\infty} \subset$ $(0, \infty)$ such that $d\left(\hat{\phi}_{k_{i}}, \hat{\psi}_{\hat{k}_{i}}\right)<2^{-i} \beta$ and $\lim _{i \rightarrow \infty} d\left(\hat{\phi}_{k_{i}}, M_{1}\right)=0$. Similarly, there is a decreasing sequence $\left\{\hat{l}_{i}\right\}_{i=0}^{\infty} \subset(-\infty, 0)$ and an increasing sequence $\left\{l_{i}\right\}_{i=0}^{\infty} \subset(0, \infty)$ such that $d\left(\hat{\phi}_{l_{i}}, \hat{\psi}_{\hat{l}_{i}}\right)<2^{-i} \beta$ and $\lim _{i \rightarrow \infty} d\left(\hat{\phi}_{l_{i}}, M_{2}\right)=0$.

Denote by $\gamma$ the following two-sided sequence:

$$
\begin{gathered}
\gamma=\left\{\gamma_{n}\right\}_{n \in \mathbb{Z}}=\left\{\hat{\psi}_{i}\right\}_{i=-\infty}^{\hat{k}_{1}-1} \cup \bigcup_{j=1}^{\infty}\left(\left\{\hat{\phi}_{i}\right\}_{i=k_{j}}^{l_{j}-1} \cup\left\{\hat{\psi}_{i}\right\}_{i=\hat{l}_{j}}^{\hat{k}_{j+1}-1}\right) \\
=\left\{\ldots, \hat{\psi}_{\hat{k}_{1}-3}, \hat{\psi}_{\hat{k}_{1}-2}, \hat{\psi}_{\hat{k}_{1}-1}, \hat{\phi}_{k_{1}}, \ldots, \hat{\phi}_{l_{1}-1}, \hat{\psi}_{\hat{l}_{1}}, \ldots, \hat{\psi}_{\hat{k}_{2}-1}, \hat{\phi}_{k_{2}}\right. \\
\left.\ldots, \hat{\phi}_{l_{2}-1}, \hat{\psi}_{\hat{l}_{2}}, \ldots, \hat{\psi}_{\hat{k}_{3}-1}, \hat{\phi}_{k_{3}}, \ldots\right\} .
\end{gathered}
$$

Observe that $\gamma$ is a two-sided limit $\beta$-pseudo-orbit. Now, let $\left\{z_{n}\right\}_{n \in \mathbb{Z}}$ be a 0 -pseudo-orbit provided by TSLmSP to $\gamma$ and denote $z=z_{0}$. We claim that

$$
\omega_{f}(z)=M_{1} \cup M_{2} \cup \hat{\psi} \cup \hat{\phi} .
$$

First of all, let us observe that by the definition of sequences $k_{i}, l_{i}$ there are increasing sequences $n_{i}, m_{i}$ such that the following limits exist: $\lim _{i \rightarrow \infty} \gamma_{n_{i}}=$ $p \in M_{1}$ and $\lim _{i \rightarrow \infty} \gamma_{m_{i}}=q \in M_{2}$. Therefore, $M_{1} \cap \omega_{f}(z) \neq \emptyset$ and $M_{2} \cap \omega_{f}(z) \neq$ $\emptyset$ which gives $M_{1} \cup M_{2} \subset \omega_{f}(z)$. Clearly, for any $s \in \mathbb{Z}$ we can find increasing sequences $r_{i}, \hat{r}_{i}$ such that $\gamma_{r_{i}}=\hat{\phi}_{s}$ and $\gamma_{\hat{r}_{i}}=\hat{\psi}_{s}$ and hence $\hat{\phi} \cup \hat{\psi} \subset \omega_{f}(z)$.

Next, fix any increasing sequence $s_{i}$ such that $\lim _{i \rightarrow \infty} f^{s_{i}}(z)$ exists. Without loss of generality, going to a subsequence if necessary, we may assume that all points $\gamma_{s_{i}}$ are elements of exactly one of the sequences $\hat{\phi}$ or $\hat{\psi}$. Let us assume that the first possibility takes place (the second case is symmetric), that is there there exists a sequence $\hat{s}_{i}$ such that $\gamma_{s_{i}}=\hat{\phi}_{\hat{s}_{i}}$ for all $i \in \mathbb{N}$. Assume first, that the sequence $\hat{s}_{i}$ is bounded. Then, going to a subsequence again, we may assume that it is constant, say $\hat{s}_{i}=s$ for all $i$, and then $\lim _{i \rightarrow \infty} f^{s_{i}}(z)=$ $\lim _{i \rightarrow \infty} \hat{\phi}_{\hat{s}_{i}}=\hat{\phi}_{s}$. If $\hat{s}_{i}$ is unbounded, then we may assume that it is monotone and then $\lim _{i \rightarrow \infty} f^{s_{i}}(z) \in M_{1}$ or $\lim _{i \rightarrow \infty} f^{s_{i}}(z) \in M_{2}$, depending whether $\hat{s}_{i}$ is decreasing or increasing. The proof is completed.

Lemma 11. Let $(X, f)$ be a transitive dynamical system with TSLmSP and let $M_{1}, M_{2}, \ldots, M_{n} \subset X$ be minimal sets. There exist $z$ and at most countable set $Z$ such that $\omega_{f}(z)=Z \cup \bigcup_{i=1}^{n} M_{i}$. 
Proof. Let $\beta$ be provided by TSLmSP. We prove the statement of the lemma by induction on $n$. The case $n=2$ is solved by Lemma 10 . Now suppose that the lemma holds with some $n=N \geq 2$. We are going to show that it also holds with $n=N+1$.

By Lemma 10 and our assumptions, there are points $z_{1}, z_{2}$ and countable sets $Z_{1}, Z_{2}$ such that

$$
\omega_{f}\left(z_{1}\right)=Z_{1} \cup \bigcup_{i=1}^{N} M_{i}, \quad \omega_{f}\left(z_{2}\right)=Z_{2} \cup M_{1} \cup M_{N+1} .
$$

Note that $M_{1} \subset \omega_{f}\left(z_{1}\right) \cap \omega_{f}\left(z_{2}\right)$, which yields that there are increasing sequences $k_{i}, s_{i}$ such that

(1) $d\left(f^{k_{i}}\left(z_{1}\right), f^{k_{i+1}}\left(z_{1}\right)\right)<2^{-i} \beta$ and $d\left(f^{s_{i}}\left(z_{2}\right), f^{s_{i+1}}\left(z_{2}\right)\right)<2^{-i} \beta$,

(2) $d\left(f^{k_{i}}\left(z_{1}\right), f^{s_{i}}\left(z_{2}\right)\right)<2^{-i} \beta$ and $d\left(f^{k_{i}}\left(z_{1}\right), M_{1}\right)<2^{-i} \beta$.

Let $\left\{\xi_{i}\right\}_{i=0}^{\infty} \subset M_{1}$ be any sequence satisfying $f\left(\xi_{i}\right)=\xi_{i+1}$ for all $i \leq 0$ and $d\left(\xi_{1}, f^{k_{1}}\left(z_{1}\right)\right)<\beta$. Now define a two-sided limit $\beta$-pseudo-orbit $\gamma$ putting

$$
\begin{gathered}
\gamma=\left\{\gamma_{n}\right\}_{n \in \mathbb{Z}}=\left\{\xi_{i}\right\}_{i=-\infty}^{0} \cup \bigcup_{j=1}^{\infty}\left(\left\{f^{i}\left(z_{1}\right)\right\}_{i=k_{j}}^{k_{j+1}-1} \cup\left\{f^{i}\left(z_{2}\right)\right\}_{i=s_{j}}^{s_{j+1}-1}\right) \\
=\left\{\ldots, \xi_{-2}, \xi_{-1}, \xi_{0}, f^{k_{1}}\left(z_{1}\right), \ldots, f^{k_{2}-1}\left(z_{1}\right), f^{s_{1}}\left(z_{2}\right), \ldots,\right. \\
\left.f^{s_{2}-1}\left(z_{2}\right), f^{k_{2}}\left(z_{1}\right), \ldots, f^{k_{3}-1}\left(z_{1}\right), f^{s_{2}}\left(z_{2}\right), \ldots\right\} .
\end{gathered}
$$

Clearly $\gamma$ is a limit $\beta$-pseudo-orbit. Now let $\left\{z_{i}\right\}_{i \in \mathbb{Z}}$ be a 0 -pseudo-orbit which traces $\gamma$ in the limit sense, and put $z=z_{0}$. Since $\left\{\gamma_{j}\right\}_{j=0}^{\infty}$ contains infinite forward segments of both orbits of $z_{1}$ and $z_{2}$ we immediately obtain that

$$
Z_{1} \cup Z_{2} \cup \bigcup_{i=1}^{N+1} M_{i}=\omega_{f}\left(z_{1}\right) \cup \omega_{f}\left(z_{2}\right) \subset \omega_{f}(z) .
$$

But conversely, if we fix any increasing sequence $t_{i}$, then, after passing to a subsequence if necessary, there is a sequence $\hat{t}_{i}$ such that $\gamma_{t_{i}}=f^{\hat{t}_{i}}\left(z_{1}\right)$ for all $i \geq 0$ or $\gamma_{t_{i}}=f^{\hat{t}_{i}}\left(z_{2}\right)$ for all $i \geq 0$. But $\left\{\gamma_{j}\right\}_{j=0}^{\infty}$ consists of blocks $f^{k_{i}}\left(z_{1}\right), \ldots, f^{k_{i+1}-1}\left(z_{1}\right)$ separated by blocks $f^{s_{i}}\left(z_{2}\right), \ldots, f^{s_{i+1}-1}\left(z_{2}\right)$ which implies we may assume that $\hat{t}_{i}$ is increasing, since $s_{i}$ is increasing. But this implies that

$$
\omega_{f}(z) \subset \omega_{f}\left(z_{1}\right) \cup \omega_{f}\left(z_{2}\right)
$$

finishing the proof.

Theorem 12. Let $(X, f)$ be a transitive but not minimal dynamical system with TSLmSP. Then there exists a c-dense $\omega$-scrambled set $Q \subset X$.

Proof. It can be proved that transitive system with shadowing is either sensitive or equicontinuous [20]. But it is easy to see that if we take a minimal system $M \subset X$ and $(X, f)$ is equicontinuous, then $X=M$, hence $(X, f)$ is sensitive. 
By results of [11] we know that transitive map with limit shadowing property (a one-sided version of TSLmSP) has SP.

By Theorem 5.2 in [22], for every nonempty open set $U$ there is $m>0$, a closed set $\Lambda \subset U$ invariant for $f^{m}$ and a factor map $\pi:\left(\Lambda, f^{m}\right) \rightarrow\left(\Sigma_{2}^{+}, \sigma\right)$. Since $\Sigma_{2}^{+}$contains an uncountable family of disjoint minimal and infinite sets (thus uncountable), there exist an uncountable set $C_{U}$ and pairwise disjoint minimal sets $\hat{M}_{c} \subset \Lambda, c \in C_{U}$ for the dynamical system $\left(\Lambda, f^{m}\right)$. Note that for each $c$ the set $M_{c}=\bigcup_{i=0}^{\infty} f^{i}\left(\hat{M}_{c}\right)=\bigcup_{i=0}^{m-1} f^{i}\left(\hat{M}_{c}\right)$ is minimal and if for some $c, d \in C_{U}$ and $i \geq 0$ we have $f^{i}\left(\hat{M}_{c}\right) \cap \hat{M}_{d} \neq \emptyset$, then $f^{i}\left(\hat{M}_{c}\right)=\hat{M}_{d}$. Therefore, if we define $c \approx d$ when $f^{i}\left(\hat{M}_{c}\right)=\hat{M}_{d}$ or $f^{i}\left(\hat{M}_{d}\right)=\hat{M}_{c}$ for some $i \geq 0$, then $\approx$ is an equivalence relation on $C_{U}$ with finite equivalence classes. This implies that we may assume (selecting exactly one element of each class) that minimal sets $M_{c}$ are pairwise disjoint for every $c \in C_{U}$. By the construction each set $M_{c}$ is uncountable.

Let us select a countable basis $\left\{U_{i}\right\}_{i=1}^{\infty}$ of the topology on $X$ and for each $U_{i}$ let us perform the above construction to obtain a family of minimal sets $M_{c}$ with $c \in C_{U_{i}}$, where each $C_{U_{i}}$ is uncountable. We claim that there are uncountable sets $D_{i} \subset C_{U_{i}}$ such that $M_{c} \cap M_{d}=\emptyset$ provided that $c \in D_{i}$, $d \in D_{j}$ and $i \neq j$.

First, for each $i$ denote $C_{i}^{(1)}=C_{U_{i}}$. Up to a bijection (not necessarily continuous), we can identify $C_{1}^{(1)}$ with $[0,1]^{2}$. Put $W=\emptyset$. Now suppose that for some $r \in[0,1]$ there is $j>1$ such that the set of indexes $c \in C_{j}^{(1)}$ such that $M_{c} \neq M_{(r, d)}$ for all $d \in[0,1]$ is at most countable (i.e., all but countably many minimal sets with indexes in $C_{j}^{(1)}$ are in the fibre of $C_{1}^{(1)}$ at the level $r$ ). In such a case we include $r$ in $W$. We perform this operation for each $j=2,3, \ldots$. As a result of this (infinite) procedure we obtain at most countable set $W$ such that if we fix any $s \in[0,1] \backslash W$, then the set of $c \in C_{j}^{(1)}$ such that $M_{c} \neq M_{(s, d)}$ for all $s \in[0,1]$ is uncountable for each $j>1$. Fix an index $s \in[0,1] \backslash W$ and denote $D_{1}=\{s\} \times[0,1] \subset C_{1}^{(1)}$. Now, if we remove from each $C_{j}^{(1)}$ indexes defining set $M_{c}$ for some $c \in D_{1}$, and denote the result of this operation by $C_{j}^{(2)}$, for each $j>1$, then by our construction every set $C_{j}^{(2)}$ is uncountable. We can perform this construction inductively, using family $\left\{C_{j}^{(n)}\right\}_{j \geq n}$ for the construction of $D_{n}$ the same way as it was done with $D_{1}$. This proves the claim.

Finally, we may pick a minimal set $M$ and guarantee that it is disjoint with all $M_{c}, c \in D=\bigcup_{i>0} D_{i}$ (e.g. we can remove one set from $\left.D_{1}\right)$. Now, for each $c \in D$ we use Lemma 10 to construct a point $z_{c}$ such that $M_{c} \cup M \subset \omega_{f}\left(z_{c}\right)$ and $\omega_{f}\left(z_{c}\right) \backslash M_{c} \cup M$ is at most countable (and in particular $z_{c} \neq z_{d}$ ). This implies that $M_{d} \cap \omega_{f}\left(z_{c}\right)=\emptyset$ for each $c \neq d$ since $M_{d}$ is uncountable and disjoint with $M_{c}$ and $M$. We may also assume (replacing $z_{c}$ by its forward iterate) that $z_{c} \in U_{i}$ for each $c \in D_{i}$ because $U_{i} \cap M_{c} \neq \emptyset$ for every $c \in D_{i}$. 
Denote $Q=\left\{z_{c}: c \in \bigcup_{i=1}^{\infty} D_{i}\right\}$. Then $Q$ is $c$-dense simply by the definition. But now, if we fix any distinct $x, y \in Q$, then there are distinct $c, d$ such that $\omega_{f}(x)=M \cup M_{c} \cup Z_{1}$ and $\omega_{f}(y)=M \cup M_{d} \cup Z_{2}$, where $Z_{1}$ and $Z_{2}$ are at most countable. In particular $M_{d} \cap \omega_{f}(x)=\emptyset$ and $M_{c} \cap \omega_{f}(y)=\emptyset$. This shows that $x, y$ form an $\omega$-scrambled pair completing the proof.

It seems that there is no chance to expect that the set of all $\omega$-scrambled pairs is $G_{\delta}$ in $X \times X$. So in practice there is no chance to use standard arguments of construction of Mycielski set. From that point of view the following question can be of interest.

Question 1. Is it possible to construct set $Q$ in Theorem 12 in such a way that it is Borel?

By standard argument (e.g. see [28]), a positive answer to the above question immediately implies that there is a dense Mycielski $\omega$-scrambled set in the system.

\section{Topological graphs and $\boldsymbol{\omega}$-chaos}

The aim of this section is to extend the results of [29] (proved for interval maps) onto the class of maps on topological graphs. While the results are more general, the proof is somehow shorter (which does not mean elementary).

The following fact completes [29, Proposition 1]. Among other things it allows to transfer Cantor $\omega$-scrambled set from higher iterate $f^{m}$ to $f$. Note that $\omega(x, f)=\bigcup_{i=0}^{n-1} \omega\left(f^{i}(x), f^{n}\right)=\bigcup_{i=0}^{n-1} f^{i}\left(\omega\left(x, f^{n}\right)\right)$ and then (under certain conditions) in an $\omega$-scrambled set $S$ for $f^{m}$, there are at most $m$ points $p, q \in S$ such that $\omega(p, f) \backslash \omega(q, f)$ is at most countable and we can define an equivalence relation in $S \times S$ with uncountably many equivalence classes (see the proof of Theorem 3.5 in [15]). This relation, however, is not necessarily Borel, hence we cannot ensure that we can pick elements of these classes in such a way that a perfect set is obtained. Therefore we need to perform a little more delicate construction, to remove this difficulty. For this purpose, we are going to adjust the argument in the proof of [29, Proposition 1].

Theorem 13. Let $(X, f)$ be a dynamical system and let $\Lambda$ and $m>0$ be such that $\left(\Lambda, f^{m}\right)$ is conjugated with $\left(\Sigma_{2}^{+}, \sigma\right)$. Then there exists a Cantor set Csubset $\Lambda$ which is $\omega$-scrambled for $f$.

Proof. First, we will perform a construction from [29, Proposition 1] to show that there exists a perfect set $S$ subset $\Sigma_{2}^{+}$which is $\omega$-scrambled for $\sigma$ and satisfies the following conditions:

(1) $0^{\infty} \in \omega(x, \sigma)$ for every $x \in S$.

(2) $\omega(x, \sigma)=M_{x} \cup V_{x} \cup U_{x} \cup\left\{0^{\infty}\right\}$ where $V_{x}, U_{x}$ are at most countable sets and $M_{x}$ is a minimal set (and all these sets depend on $x$; are pairwise disjoint). 
(3) for every $\varepsilon>0$ there is $\delta>0$ such that if $d(x, y)<\delta, x, y \in S$, then $\mathcal{H}_{d}\left(M_{x}, M_{y}\right)<\varepsilon$, where $\mathcal{H}_{d}$ is the Hausdorff metric induced by $d$.

Denote by $A \subset \Sigma_{2}^{+}$the set containing all sequences with infinitely many occurrences of symbols zero and one, that is $a \in A$ provided that both sets $\left\{i: a_{i}=0\right\}$ and $\left\{i: a_{i}=1\right\}$ are infinite. Note that $\Sigma_{2}^{+} \backslash A$ is at most countable, and hence $A$ is a Borel set. Then there is a Cantor set $\hat{A} \subset A \subset \Sigma_{2}^{+}$(see [28]). Denote by $\mathcal{N}$ a partition of $\mathbb{N}$ by the following sets $N_{n}=2^{n-1}\left(1+2 \mathbb{N}_{0}\right)$, e.g. $N_{1}=\{1,3,5, \ldots\}, N_{2}=\{2,6,10, \ldots\}$, etc. Using the partition $\mathcal{N}$, we define a map $\Phi: \Sigma_{2}^{+} \rightarrow \Sigma_{2}^{+}$putting $\Phi(x)_{i}=x_{n}$ where $n$ is the unique index such that $i \in N_{n}$. Roughly speaking, $\Phi(x)$ has the following structure

$$
\Phi(x)=x_{1} x_{2} x_{1} x_{3} x_{1} x_{2} x_{1} x_{4} x_{1} x_{2} x_{1} x_{3} x_{1} x_{2} x_{1} x_{5} x_{1} \cdots .
$$

Clearly $\Phi$ is continuous and one-to-one, hence the set $D=\Phi(\hat{A})$ is a Cantor set. For every $\beta \in D$ denote $D_{\beta}=\omega(\beta, \sigma)$. It follow directly by the construction that $D_{\beta}$ is a minimal set and $\beta \in D_{\beta}$. Note that if $x \in D_{\beta}$, then $x_{n}=\beta_{1}$ for all $n \in N_{1}$ or all $n \in N_{1}+1$ and if $x_{n}=\beta_{1}$ for all $n \in N_{1}$, then we can find $i, j \in N_{1}+1$ such that $x_{i} \neq x_{j}$ (or $i, j \in N_{1}$ in the second possible case). We can repeat this observation for $N_{2}, N_{3}$, etc. showing that $D_{\beta} \neq D_{\alpha}$ when $\beta \neq \alpha$.

Note that if for some $k>0$ we define $\Phi_{k}(x)_{i}=x_{n}$ where $n$ is the unique index such that $i \in N_{n}$ and $n \leq k$ and $\Phi_{k}(x)=*$ otherwise, where $*$ is an additional symbol other than 0,1 , then $\Phi_{k}(x)_{[i, i+k]}$ contains at most one occurrence of $*$. Furthermore, observe that if we fix any $i$ and put 0 or 1 in place of $*$ in the word $w=\Phi_{k}(x)_{[i, i+k]}$, then $w$ can be located somewhere in $\Phi(x)$. This implies that if $x_{[1, k]}=y_{[1, k]}$, then $B_{k+1}\left(D_{\Phi(x)}\right)=B_{k+1}\left(D_{\Phi(y)}\right)$ and hence $\mathcal{H}_{d}\left(D_{\Phi(x)}, D_{\Phi(y)}\right) \leq 2^{-k}$.

Let $\beta=\Phi(x)$ and define

$$
\Psi(x)=\beta_{1} 0 \beta_{1} \beta 200 \beta_{1} \beta_{2} \beta_{3} 000 \beta_{1} \cdots \beta_{[1, n]} 0^{n} \cdots .
$$

Denote $U_{x}=\left\{\beta_{[1, n)} 0^{\infty}: n \geq 1\right\}$ and $V_{x}=\left\{0^{n} \beta: n \geq 1\right\}$. Then $U_{x}, V_{x}$ are countable sets and $\omega(\Psi(x), \sigma)=\left\{0^{\infty}\right\} \cup U_{x} \cup V_{x} \cup M_{\Phi(x)}$, e.g. by Lemma 2.2 in [15]. Combining all the previous remarks together and putting $S=\Phi(\hat{A})$ we obtain that conditions (1)-(3) are satisfied.

Since $\left(\Lambda, f^{m}\right)$ is conjugated with $\left(\Sigma_{2}^{+}, \sigma\right)$, without loss of generality we may assume that $S \subset \Lambda$. For every $x \in X$ denote $K_{x}=\bigcup_{i=0}^{\infty} f^{i}\left(M_{x}\right)=$ $\bigcup_{i=0}^{m-1} f^{i}\left(M_{x}\right)$. Denote by $R$ the set of pairs in $S \times S$ that are $\omega$-scrambled.

This implies that for every $x \in S$ there are at most $m$ other points $z \in S$ such that $K_{x}=K_{y}$. Clearly, for each $x \in S$ the set $K_{x}$ is minimal and $\omega(x, f) \backslash K_{x}$ is at most countable. By (3), for every $\varepsilon>0$ there is $\delta$ such that if $K_{x} \cap K_{y}=\emptyset$, then $K_{p} \cap K_{q}=\emptyset$ provided that $p \in B(x, \delta) \cap S$ and $q \in B(y, \delta) \cap S$. This proves that $R$ is an open set. But if we fix any distinct $x, y \in S$ and any open neighborhood $U$ of $x$, then $U \cap S$ is infinite, and so there is $z \in U \cap S$ such that $K_{z} \cap K_{y}=\emptyset$. This proves that the pair $(z, y)$ is $\omega$-scrambled and therefore $R$ 
is a residual subset of $S \times S$. By Lemma 1 there is a Cantor set $C \subset S$ such that $C \times C \subset R \cup \Delta$. The proof is completed.

The following fact was proved in [21] for interval maps, however the proof is the same in the context of graph maps. The only difference is that at first we have to use [16] to find a horseshoe for an iterate of map with positive entropy.

Lemma 14. Let $(G, f)$ be a dynamical system with positive topological entropy acting on a topological graph $G$. Then there exist $n>0$, an $f^{n}$-invariant closed set $\Lambda$ and a homeomorphism $\pi: \Lambda \rightarrow \Sigma_{2}^{+}$such that $\pi \circ\left(\left.f^{n}\right|_{\Lambda}\right)=\sigma \circ \pi$.

Lemma 15. Let $G$ be a topological graph and let $(G, f)$ be a topologically mixing dynamical system. Then there exists a dense $\omega$-chaotic Mycielski set.

Proof. It is well known that $(G, f)$ has positive topological entropy (see [8] for proof of this fact in much wider context). Let $\Lambda$ and $n$ be provided by Lemma 14. By Theorem 13 there is an $\omega$-scrambled Cantor set $C$ for $(G, f)$.

By Theorem 4.6 in $[10]$ there is a finite set $I(f)$ such that for every nonempty open set $U$ and every $\varepsilon>0$ there is $n>0$ such that $f^{n}(U) \supset G \backslash B(I(f), \varepsilon)$. Since $I(f)$ is finite, without loss of generality we may assume that $C \cap I(f)=\emptyset$ and hence there is $\gamma>0$ such that $C \cap B(I(f), \gamma)=\emptyset$. Therefore, for every nonempty open set $U$ there is $n>0$ such that $C \subset f^{n}(U)$.

Since $C$ is homeomorphic with $C \times C$, there exist a sequence of pairwise disjoint Cantor sets $C_{1}, C_{2}, \ldots \subset C$. If $V$ is an open set and $n>0$ is such that $C_{i} \subset f^{n}(\bar{V})$, then $A=f^{-n}\left(C_{i}\right) \cap \bar{V}$ is compact, and hence by [28, Remark 4.3.6] there is a Cantor set $D_{i} \subset \bar{V}$ such that $f^{n}$ is injective on $D_{i}$ and $f^{n}\left(D_{i}\right) \subset C_{i}$. Fix any base $U_{1}, U_{2}, \ldots$ of the topology of $X$ and open sets $V_{i}$ such that $\overline{V_{i}} \subset U_{i}$. For every $i$ there is $n$ such that $C_{i} \subset C \subset f^{n}\left(V_{i}\right) \subset f^{n}\left(\overline{V_{i}}\right)$ and as a result there is also a Cantor set $D_{i}$ such that $f^{n}\left(D_{i}\right) \subset C_{i}$ and if we fix distinct $x, y \in D_{i}$, then $f^{n}(x), f^{n}(y) \in C_{i}$ are also distinct. Clearly for every $x \in X$ and $n>0$ we have $\omega(x, f)=\omega\left(f^{n}(x), f\right)$, thus $M=\bigcup_{i=1}^{\infty} D_{i}$ is a Mycielski $\omega$-scrambled set. But $\emptyset \neq M \cap \overline{V_{i}} \subset M \cap U_{i}$ which proves that $M$ is also dense, completing the proof.

Theorem 16. Let $G$ be a topological graph. If $(G, f)$ is transitive dynamical system other than irrational rotation, then there exists a dense Mycielski $\omega$ chaotic set for $f$.

Proof. The only examples of transitive graph homeomorphisms are the irrational rotations of the circle, hence we may assume that $(G, f)$ is not invertible. By periodic decomposition theorem (see [3] or [1]) there are graphs $G_{0}, \ldots, G_{s-1} \subset G$ with disjoint interiors, such that $f\left(G_{i}\right)=G_{i+1(\bmod s)}$ and $\left.f^{s}\right|_{G_{i}}$ is totally transitive (hence mixing, see [2, 4] or [9] for a proof).

By Lemma 15 there is a dense Mycielski set $M \subset G_{0}$ for $\left.f^{s}\right|_{G_{0}}$. Graphs $G_{i}$ have disjoint interiors, hence without loss of generality we may assume that $M \cap G_{i}=\emptyset$ for each $i \neq 0$. Since $M$ is a dense Mycielski set, it is easy to find disjoint dense Mycielski sets $M_{0}, \ldots, M_{s-1} \subset M$. Additionally each $M_{i}$ is dense 
and $f^{i}\left(G_{0}\right)=G_{i}$, hence the set $f^{i}\left(M_{i}\right)$ is dense in $G_{i}$. But $M_{i}$ is scrambled set, thus $\left.f\right|_{M_{i}}$ is one-to-one. This proves that $f^{i}\left(M_{i}\right)$ is Mycielski set and so also $S=\bigcup_{i=0}^{s-1} f^{i}\left(M_{i}\right)$ is a dense Mycielski set. The proof is complete.

Acknowledgements. Research leading to the results contained in this paper was supported by Narodowe Centrum Nauki (National Science Center) in Poland, grant no. DEC-2011/03/B/ST1/00790. The author was also supported by the Polish Ministry of Science and Higher Education (AGH local grant). Financial support of these institutions is gratefully acknowledged.

\section{References}

[1] Ll. Alsedà, M. A. del Río, and J. A. Rodríguez, A splitting theorem for transitive maps, J. Math. Anal. Appl. 232 (1999), no. 2, 359-375.

[2] _ Transitivity and dense periodicity for graph maps, J. Difference Equ. Appl. 9 (2003), no. 6, 577-598.

[3] J. Banks, Regular periodic decompositions for topologically transitive maps, Ergodic Theory Dynam. Systems 17 (1997), no. 3, 505-529.

[4] J. Banks and B. Trotta, Weak mixing implies mixing for maps on topological graphs, J. Difference Equ. Appl. 11 (2005), no. 12, 1071-1080.

[5] J. Bobok, On multidimensional w-chaos, Internat. J. Bifur. Chaos Appl. Sci. Engrg. 16 (2006), no. 3, 737-740.

[6] B. Carvalho, Hyperbolicity, transitivity and the two-sided limit shadowing property, Proc. AMS, to appear.

[7] B. Carvalho and D. Kwietniak, On homeomorphisms with the two-sided limit shadowing property, preprint, arXiv:1402.0674.

[8] M. Dirbák, L. Snoha, and V. Špitalský, Minimality, transitivity, mixing and topological entropy on spaces with a free interval, Ergodic Theory Dynam. Systems 33 (2013), no. $6,1786-1812$

[9] G. Harańczyk, D. Kwietniak, and P. Oprocha, A note on transitivity, sensitivity and chaos for graph maps, J. Difference Equ. Appl. 17 (2011), no. 10, 1549-1553.

[10] _ , Topological structure and entropy of mixing graph maps, Ergodic Theory Dynam. Systems (2013); doi: 10.1017/etds.2013.6

[11] M. Kulczycki, D. Kwietniak, and P. Oprocha, On almost specification and average shadowing properties, preprint, 2013.

[12] D. Kwietniak and P. Oprocha, A note on the average shadowing property for expansive maps, Topology Appl. 159 (2012), no. 1, 19-27.

[13] M. Lampart and P. Oprocha, Shift spaces, $\omega$-chaos and specification property, Topology Appl. 156 (2009), no. 18, 2979-2985.

[14] K. Lee and K. Sakai, Various shadowing properties and their equivalence, Discrete Contin. Dyn. Syst. 13 (2005), no. 2, 533-540.

[15] S. H. Li, w-chaos and topological entropy, Trans. Amer. Math. Soc. 339 (1993), no. 1, 243-249.

[16] J. Llibre and M. Misiurewicz, Horseshoes, entropy and periods for graph maps, Topology 32 (1993), no. 3, 649-664.

[17] G. Lu, K. Lee, and M. Lee, Generic diffeomorphisms with weak limit shadowing, Adv. Difference Equ. 2013 (2013), article id: 27, doi: 10.1186/1687-1847-2013-27.

[18] J. H. Mai and X. Ye, The structure of pointwise recurrent maps having the pseudo orbit tracing property, Nagoya Math. J. 166 (2002), 83-92.

[19] J. Mycielski, Independent sets in topological algebras, Fund. Math. 55 (1964), 137-147. 
[20] T. K. S. Moothathu, Implications of pseudo-orbit tracing property for continuous maps on compacta, Top. Appl. 158 (2011), no. 16, 2232-2239.

[21] _ Syndetically proximal pairs, J. Math. Anal. Appl. 379 (2011), no. 2, 656-663.

[22] T. K. S. Moothathu and P. Oprocha, Shadowing entropy and minimal subsystems, Monatsh. Math. 172 (2013), no. 3-4, 357-378.

[23] K. Palmer, Shadowing in Dynamical Systems, Kluwer Academic Publishers, Dordrecht, 2000.

[24] R. Pikuła, On some notions of chaos in dimension zero, Colloq. Math. 107 (2007), no. 2, $167-177$.

[25] S. Yu. Pilyugin, Shadowing in Dynamical Systems, Springer-Verlag, Berlin, 1999.

[26] L Sets of dynamical systems with various limit shadowing properties, J. Dynam. Differential Equations 19 (2007), no. 3, 747-775.

[27] K. Sakai, Diffeomorphisms with the s-limit shadowing property, Dyn. Syst. 27 (2012), no. $4,403-410$

[28] S. M. Srivastava, A Course on Borel Sets, Graduate Texts in Mathematics, vol. 180, Springer-Verlag, New York, 1998.

[29] J. Smítal and M. Štefánková, Omega-chaos almost everywhere, Discrete Contin. Dyn. Syst. 9 (2003), no. 5, 1323-1327.

AGH University of Science and Technology Faculty of Applied Mathematics

al. Mickiewicza 30, 30-059 Kraków, Poland

AND

Institute of Mathematics

Polish ACAdemy of Sciences

UL. Śniadeckich 8, 00-956 Warszawa, Poland

E-mail address: oprocha@agh.edu.pl 